DETONATION AND TWO-PHASE FLOW

\title{
PER TURBATION ANALYSIS OF ONE-DIMENSIONAL HETEROGENEOUS FLOW IN ROCKET NOZZLES
}

\author{
W. D. Rannie \\ California Institute of Technology, Pasadena, Calif.
}

\section{ABSTRACT}

A perturbation analysis of the velocity and temperature lags in two-phase flow in rocket nozzles is developed and applied to the calculation of specific impulse and otier performance characteristics of nozzles of arbitrary shape. Within the limitations of the one-dimensional flow approximation, the analysis is valid for distributions of particle diameters that are in a practical range.

\section{INTRODUCTION}

The development of high energy propellants, with products of combustion partially in the form of finely divided particles, has increased interest in the effects of the heterogenecus flows on performance. The particles, swept through the nozzle by gas flow, lag behind the gas in temperature drop as well as velocity increase, both lags degrading performance and partially ciefeating the purpose of the high energy propellants.

Analysis of the heterogeneous flow in the rocket nozzle is necessarily complex because of the large number of separate parameters required to specify particle characteristics and the nozzle contour. Most previous investigations (e. 3., Refs. 1-4) have required numerical procedures at an early stage of the analysis, thus tending to conceal the influence of individual parameters and making necessary a great number of specific calculations. Clearly an analytical solution of the problem, even if complex in form, has the advantage that the

W. D. RANNIE is Robert H. Goddard Professor of Jet Propulsion, Daniel and Florence Guggenheim Jet Propulsion Center, Kármán Laboratory of Fluid Mechanics and Jet Propulsion. Submitted for publication to the American Rocket Society, Jan. 3, 1962. 


\section{DETONATION AND TWO-PHASE FLOW}

influence of each parameter can be traced relatively easily.

The perturbation procedure developed here is confined to the one-dimensional hydraulic approximation, which is known to be satisfactory for the flow of a homogeneous fluid in a nozzle. The magnitude of the error that is introduced with the same approximation for heterogeneous flow is difficult to estimate, since the boundary conditions appropriate to particles impinging on the nozzle wall are not known. The additional simplifying assumptions, that the particle conductivity is very high compared with the gas and that radiative heat transfer between particles is small, compromise the solution much less seriously.

The perturbation solution requires that velocity and temperature lags be small compared with the velocity and temperature of the unperturbed flow without lag. Although this requirement introduces some restrictions on nozzle shape and particle size, it appears that the method will be valid (as far as the one-dimensional analysis can be valid) for most practical applications. For instance, the error in the perturbation solution ordinarily will be small for particle diameters up to $5 \times 10^{-4} \mathrm{~cm}$, the upper limit depending on nozzle dimensions and shape as well as on the stagnation conditions in the rocket chamber. The range of size of particles under actual operating conditions is not yet firmly established; however, the evidence at hand indicates that the diameters of most of the particles will not exceed the forementioned figure.

The perturbation analysis is formulated in terms of pressure as the independent variable. This choice of independent variable has advantages that do not seem to have been exploited sufficiently in the previous investigations. With pressure as the independent variable, the dependence of nozzle cross-sectional area on axial distance does not appear explicitly in the unperturbed solution with no lag, and hence the area can be chosen arbitrarily in the perturbed solution. With axial distance as the independent variable, the usual choice in earlier investigations, the cross-sectional area appears in the solution without lag as well as in the solution with lag. When lags are introduced, the area dependence on axial distance must be changed to satisfy required throat conditions. Hence direct comparison of the same nozzle shape with and without lag is not possible. With pressure as the independent variable this difficulty does not arise.

The major complication in the analysis is evaluation of the drag and heat transfer between particles and gas. In the Stokes' regime of flow, this is quite simple, but particles 
DETONATION AND TWO-PHASE FLOW

satisfying the conditions for Stokes' flow have negligible lags. When the lags are appreciable, the Reynolds number of the relative flow becomes large in the neighborhood of the nozzle throat, and slip flow phenomena become important near the nozzle exit. Both of these effects have strong influence on drag and heat transfer and increase the difficulty of scaling to different conditions and dimensions.

In the sections following, the equations governing the one-dimensional heterogeneous flow are established (cf., also Refs. 3-4) and are put in the most suitable form for perturbation analysis. Solutions for the first and second approximations are obtained, where the first approximation corresponds to flow with no lag. General expressions are derived for the second approximations to the mass flow rate, specific impulse, and thrust for an arbitrary nozzle shape in terms of a single numerical integration. The parameter defining the nozzle contour and the correction parameters for drag and heat transfer outside the Stokes' regime are discussed in some detail. A specific example for particles of a single diameter illustrates the method and forms a basis for estimation of the range of validity and certain general conclusions. A simple means of extending the analysis to a distribution of particle size is derived.

\section{GOVERNING EQUATIONS}

Let $\alpha$ be the mass fraction of particles in the heterogeneous mixture, $\rho_{g}$ the gas density, $p_{s}$ the density of the solid (or liquid) material of the particles, and $\rho$ the density of the mixture. Then

$$
\frac{1}{\rho}=\frac{1-\alpha}{\rho g}+\frac{\alpha}{\rho_{s}}
$$

where $(1-\alpha) \rho$ is the density of gas per unit volume of mixture and $\alpha \rho$ is the density of particles per unit volume of mixture. The mass fraction of particles in the mixture. when both gas and particles have the same velocity (i.e., no lag) will be denoted $\alpha_{0}$. In general, $\alpha=\alpha_{0}$ only at stagnation conditions in the rocket chamber.

Let $A$ denote the area of cross section of the nozzle at any point along its axis, and let $\dot{m}$ be the constant mass flow rate of the mixture. Then the conservation of mass flow rates for gas and particles separately lead to the equations

$$
(1-\alpha) \rho u_{g} A=\left(1-\alpha_{0}\right) \stackrel{\circ}{m}
$$

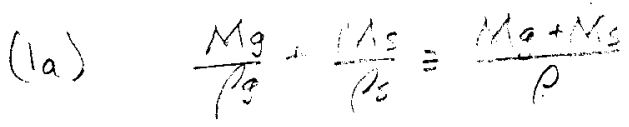


DETONATION AND TWO-PHASE FLOW

$$
\alpha \rho u_{s} A=\alpha_{0} \dot{m}
$$

where $u_{g}$ is the velocity of the gas and $u_{s}$ is the velocity of the particles. All particles are assumed to be of the same size and to have the same velocity at any cross section.

The momentum equation for the mixture, neglecting friction on the walls, is

$$
\frac{d}{d x}\left[(1-\alpha) \rho u_{g} A u_{g}\right]+\frac{d}{d x}\left[\alpha \rho u_{s} A u_{s}\right]=-\frac{d}{d x}(p A)+p \frac{d A}{d x}
$$

where $p$ is the pressure in the gas and $x$ is the distance along the nozzle axis, positive downstream. Applying Eqs. 2 and 3 , the momentum equation becomes

$$
(1-\alpha) \rho u_{g} \frac{d u_{g}}{d x}+\alpha \rho u_{s} \frac{d u_{s}}{d x}+\frac{d b}{d x}=0
$$

This equation is quite general, whatever the force interaction between particles and gas.

The energy equation for steady flow of a homogeneous fluid with no heat addition is

$$
\rho A u\left(e+\frac{1}{2} u^{2}\right)+A p u=\text { constant }
$$

where $e$ is the internal energy per unit mass. The corresponding equation for the mixture is

$$
(1-\alpha) \rho A u_{g}\left(e_{g}+\frac{1}{2} u_{g}^{2}\right)+\alpha \rho A u_{s}\left(e_{s}+\frac{1}{2} u_{s}^{2}\right)+A p\left[u_{g}^{(1-\alpha) \rho} \frac{\alpha \rho}{\rho_{g}}+u_{s} \frac{\alpha \rho}{\rho_{s}}\right]=\dot{E}_{c}[5]
$$

where $\dot{E}_{c}$ is the total rate of energy flow from the chamber. The rate of working of the pressure force per unit area ( pu for the homogeneous fluid) must be weighted by the ratio of component volume to mixture volume when applied to components of the mixture. Again, this equation is quite general, whatever the force interaction and heat transfer processes between particles and gas.

The particles will be approximated by small spheres of radius $a$, and the drag law will be based on Stokes' formula with a correction factor to allow for conditions outside the Stokes' regime of flow. Assuming that the steady state drag law is applicable to the accelerated motion, the force balance for a single particle is represented by 


$$
\frac{4}{3} \pi a^{3} \rho_{s} u_{s} \frac{d u_{s}}{d x}=6 \pi a \mu\left(u_{g}-u_{s}\right) \frac{1}{f_{d}}-\frac{4}{3} \pi a^{3} \frac{d p}{d x}
$$

where $\mu$ is the viscosity of the gas. The second term on the right is the contribution of the pressure gradient in the gas. The factor $f_{d}$ is the correction factor to the drag and, in general, is a function of Reynolds number and Mach number of the relative flow; $f_{d}=1$ in the Stokes' regime. Eq. $6 a$ can be written in more convenient form as

$$
u_{g}-u_{s}=\frac{2}{q} \frac{a^{2} p_{s}}{\mu} f_{d}\left(u_{s} \frac{d u_{s}}{d x}-\frac{1}{\rho_{s}} \frac{d p}{d x}\right)
$$

In the range of Reynolds number where Stokes' formula is applicable, heat is transferred primarily by conduction. The rate at which heat is conducted from a sphere of radius $a$ and temperature $T_{s}$ to a fluid at temperature $T_{g}$ far from the sphere is $k\left(T_{s}-T_{g}\right) / a$ per unit area, where $k$ is the gas conductivity. Hence the heat balance for a single particle is

$$
\frac{4}{3} \pi a^{3} \rho_{s} c u_{s} \frac{d T_{s}}{d x}=-\frac{k}{a}\left(T_{s}-T_{g}\right) 4 \pi a^{2} \frac{1}{f_{h}}
$$

where $C$ is the specific heat of the particle material. The factor $f_{h}$ is a correction factor similar to $f_{d}$ and depends on Reynolds number and Mach number in much the same way as $f_{d}$. Rearranging Eq. $7 a$

$$
T_{g}-T_{s}=\frac{1}{3} \frac{a^{2} \rho_{s}}{k} C f_{k} u_{s} \frac{d T_{s}}{d x}
$$

In most applications the volume occupied by the particles is much smaller than the volume occupied by the gas and can be neglected in comparison. The gas density at stagnation conditions will seldom be larger than $0.4 \mathrm{lb} / \mathrm{ft}^{3}$; if the particle material has a density of $240 \mathrm{lb} / \mathrm{ft}^{3}$, for instance, the error in the approximation

$$
\rho_{g} \cong(1-\alpha) \rho
$$

to Eq. 1 is less than one percent with a particle mass fraction $\alpha$ as high as 0.8 . Correspondingly small errors are introduced by dropping the second term in the brackets in Eq. 5 and the second term in parentheses in Eq. $6 \mathrm{~b}$.

The variable particle mass fraction $\alpha$ may be eliminated from the equations with the relation

$$
\alpha u_{s}=(1-\alpha) u_{g} \frac{\alpha_{0}}{1-\alpha_{0}}
$$

obtained from Eqs. 2 and 3. From Eq. 9 and the approximations introduced by Eq. 8, the equations for conservation of 
mass, momentum, and energy (i.e., Eqs. 2, 4, and 5) become, respectively

$$
\begin{gathered}
\rho_{g} u_{g} A=\left(1-\alpha_{0}\right) \dot{m} \\
u_{g} \frac{d u_{g}}{d x}-\alpha_{0} u_{g}\left(\frac{d u_{g}}{d x}-\frac{d u_{s}}{d x}\right)+\frac{1-\alpha_{0}}{\rho_{g}} \frac{d p}{d x}=0 \\
\left(1-\alpha_{0}\right) e_{g}+\alpha_{0} e_{s}+\frac{1}{2} u_{g}^{2}-\frac{1}{2} \alpha_{0}\left(u_{g}^{2}-u_{s}^{2}\right)+\frac{1-\alpha_{0}}{\rho_{g}} p=\frac{\dot{E}_{c}}{\dot{m}}
\end{gathered}
$$

The gas will be assumed perfect with constant specific heats; if the gas constant is $R$, then

$$
p=\pi \rho_{g} T_{g}
$$

and if $c_{v}$ and $c_{p}$ are the specific heats of the gas

$$
e_{g}+\frac{p}{\rho_{g}}=c_{v} T_{g}+R T_{g}=c_{p} T_{g}
$$

It is convenient to introduce the specific heat $c_{p_{0}}$ for the mixture with particle mass fraction $\alpha_{0}$

$$
c_{p_{0}}=\left(1-\alpha_{0}\right) c_{p}+\alpha_{0} c
$$

and a modified gas constant $R_{0}$ defined as

$$
R_{0}=\left(1-\alpha_{0}\right) T
$$

Then $\dot{E}_{c} / \dot{m}=c_{p_{0}} T_{c}$, where $T_{c}$ is the stagnation temperature for the mixture. Further, the dependent variables will be reduced to dimensionless form by the definitions

$$
u=\sqrt{\pi_{0} T_{c}} \phi \quad T=T_{c} r \quad p=p_{c} s
$$

where $p_{c}$ is the stagnation pressure in the chamber. Then Eqs. 10-12 can be written

$$
\begin{gathered}
A=\dot{m} \frac{\sqrt{R_{0} T_{c}} \frac{\tau_{g}}{p_{c}}}{\rho \phi_{g}} \\
\phi_{g} \frac{d \phi_{g}}{d \rho}+\frac{\tau_{g}}{\xi}=\alpha_{0} \phi_{g}\left(\frac{d \phi_{g}}{d \rho}-\frac{d \phi_{s}}{d g}\right) \\
\tau_{g}+\frac{1}{2} \frac{T_{0}}{c_{p_{0}}} \phi_{g}^{2}=1+\frac{1}{2} \alpha_{0} \frac{T_{0}}{c_{p_{0}}}\left(\phi_{g}^{2}-\phi_{s}^{2}\right)+\alpha_{0} \frac{c}{c_{p_{0}}}\left(\tau_{g}-T_{s}\right)
\end{gathered}
$$

In deriving Eq. 19 from Eq. 11, the independent variable was changed from $x$ to $p=p_{c} s$. There is no difficulty in doing so because $x$ appears explicitly only in Eq. 11, and there only through the factor $d x$ in the denominator.

The gas viscosity $\mu$ occurring in Eq. $6 \mathrm{~b}$ depends on the 
gas temperature and will vary appreciably through the length of the nozzle. This variation can be approximated satisfactorily by a power law of the form

$$
\mu=\mu_{c}\left(\frac{T_{g}}{T_{c}}\right)^{n}=\mu_{c} \tau_{g}^{x}
$$

where $n \cong 0.6$ for the temperature range of interest. The gas conductivity, varies in the same way, since the Prandtl number $P_{r}=\mu c_{\beta} / k$ is very nearly constant in a gas over a wide temperature range; hence it can be assumed that

$$
k=\frac{c_{p}}{P_{r}} \mu_{c} \tau_{g}{ }^{n}
$$

It is convenient to introduce a dimensionless parameter $\epsilon$ depending on particle size and stagnation conditions; this parameter is defined by the relation

$$
\epsilon=\frac{2}{q} a^{2} \frac{\rho_{s} \sqrt{R_{0} T_{c}}}{r_{t} \mu_{c}}
$$

where $Y_{t}$ is the nozzle throat radius. Defining a dimensionless length coordinate $\xi$ along the nozzle axis by

$$
x=r_{t} \xi
$$

and another dimensionless constant $\beta$ by the definition

Eqs. $6 \mathrm{~b}$ and $7 \mathrm{~b}$ become

$$
\beta=\frac{3}{2} \frac{c}{c_{p}} P_{r}
$$

$$
\begin{aligned}
& \phi_{g}-\phi_{s}=\epsilon f_{d} \frac{1}{\tau_{g}^{n}} \phi_{s} \frac{d \phi_{s}}{d g} \frac{1}{\xi^{\prime}} \\
& \tau_{g}-\tau_{s}=\epsilon \beta f_{h} \frac{1}{\tau_{g}^{n}} \phi_{s} \frac{d \tau_{s}}{d g} \frac{1}{\xi^{\prime}}
\end{aligned}
$$

where $\xi^{\prime}=d \xi / d \rho$. Since $A$ is a prescribed function of $\xi$, i.e. $A=A(\xi)$

there are now six equations (Eqs. 18-20 and 26-28) for the six unknowns $\left(\phi_{g}, \phi_{s}, \tau_{g}, \tau_{s}, A\right.$, and $\left.\xi\right)$ as functions of

\section{FIRST APPROXIMATION: ZERO VELOCITY AND TEMPER-} ATURE LAGS

An examination of Eqs. 26 and 27 shows that for $a \rightarrow 0$ (i.e., $\epsilon \rightarrow 0$ ), $\quad \phi_{g} \rightarrow \phi_{s}$ and $\tau_{g} \rightarrow \tau_{s}$, since all other factors in the equations are of order unity. In particular, $\xi$ ' is always negative, corresponding to a monotonic pressure drop through the nozzle. Hence, with subscript zero denoting zero lag, Eqs. 26 and 27 reduce to 


\section{DETONATION AND TWO.PHASE FLOW}

$$
\phi_{g}=\phi_{s}=\phi_{0} \quad \gamma_{g}=\tau_{s}=\tau_{0}
$$

for sufficiently small particles, and Eqs. 19 and 20 become

$$
\begin{aligned}
& \phi_{0} \phi_{0}^{\prime}+\tau_{0} / \rho=0 \\
& \tau_{0}+\frac{1}{2} \frac{\gamma_{0}-1}{\gamma_{0}} \phi_{0}^{2}=1
\end{aligned}
$$

The ratio of specific heats $\gamma_{0}$ is introduced by analogy with the perfect gas relation from the definition

since $c_{p_{0}}-c_{r_{0}}=R_{0}$.

$$
\frac{g_{0}}{j_{0}-1}=c_{p_{0}} / R_{0}
$$

The solution of Eqs. 30 and 31 is well known, and results are listed for future reference:

$$
\begin{aligned}
& \tau_{0}=\rho^{\frac{\gamma_{0}}{\gamma_{0}-1}} \\
& \phi_{0}=\sqrt{\frac{\gamma_{0}}{\gamma_{0}-1}}\left(1-\rho^{\frac{y_{0}-1}{\gamma_{0}}}\right)^{1 / 2} \\
& \frac{A^{\prime}}{A}=-\frac{1-\frac{r_{0}+1}{2} \rho^{\frac{r_{0}-1}{\gamma_{0}}}}{r_{0} \rho\left(1-\rho \frac{r_{0}-1}{r_{0}}\right)} \\
& \frac{A}{A_{t}}=\sqrt{\frac{g_{0}-1}{2 r_{0}}} \frac{1}{T_{0}} \frac{1}{\rho^{1 / r_{0}}\left(1-\rho \frac{r_{0}-1}{\gamma_{0}}\right)^{1 / 2}} \\
& A_{t}=\Gamma_{0} \frac{\dot{m} \sqrt{R_{0} T_{c}}}{p_{c}} \\
& \Gamma_{0}=\frac{1}{\sqrt{f_{0}}}\left(\frac{f_{0}+1}{2}\right)^{\frac{f_{0}+1}{2\left(r_{0}-1\right)}}
\end{aligned}
$$

where $A_{t}$ is the area of cross section at the throat.

SECOND APPROXIMATION: SMALI VELOCITY AND TEMPERATURE LAGS

As the radius $a$ of the particles is increased, Eqs. 26 and 27 show that the velocity difference $\phi_{g}-\phi_{s}$ and the temperature difference $\tau_{g}-\tau_{s}$ will increase in absolute value 
DETONATION AND TWO-PHASE FLOW

from zero. Expansions in powers of the parameter $\epsilon$ proportional to $a^{R}$ seem appropriate; hence it is assumed that

$$
\begin{aligned}
& \phi_{g}(s)=\phi_{0}(s)+\epsilon \phi_{g_{1}}(s)+\cdots \\
& \phi_{s}(s)=\phi_{0}(s)+\epsilon \phi_{s_{1}}(s)+\cdots \\
& \tau_{g}(s)=\tau_{0}(s)+\epsilon \tau_{g}(s)+\cdots \\
& \tau_{s}(s)=\tau_{0}(s)+\epsilon \tau_{s_{1}}(s)+\cdots \\
& \xi(s)=\xi_{0}(s)+\epsilon \xi_{1}(s)+\cdots
\end{aligned}
$$

The criterion for the validity of such expansions is that

$$
\frac{\phi_{g}-\phi_{0}}{\phi_{0}}=\epsilon \frac{\phi_{g_{1}}}{\phi_{0}}<1
$$

and corresponding inequalities for the other functions. The parameter $\epsilon$ need not be small compared with unity provided that inequalities of the type given by Eq. 40 are satisfied; that is, $\varepsilon$ is an indicator of a small quantity rather than a small quantity in its own right. The rate of convergence of the expansions in Eqs. 39 and estimates of error in truncating at any particular term can be estimated in a straightforward way when the coefficients of powers of $\epsilon$ in the expansion are themselves independent of $a$. This will be true in the Stokes' regime of flow, where $f_{d}=f_{h}=1$, but outside of this regime, $f_{d}$ and $f_{h}$ depend on $a$ and estimates of error become more involved. The analysis here is confined to the second term in the expansions above; confidence in the validity of the results is based on how well inequalities similar to Eq. 40 are satisfied.

Substituting the expansions, Eqs. 39, into Eqs. 26 and 27 and retaining only first order terms in $\epsilon$

$$
\begin{aligned}
& \phi_{g_{1}}-\phi_{s_{1}}=f_{d} \frac{1}{\tau_{0}^{n}} \phi_{0} \phi_{0}^{\prime} \frac{1}{\xi_{0}^{\prime}} \\
& \tau_{g_{1}}-\tau_{s_{1}}=\beta f_{h} \frac{1}{\tau_{0}^{n}} \phi_{0} \tau_{0}^{\prime} \frac{1}{\xi_{0}^{\prime}}
\end{aligned}
$$

With these expressions and substitution of the expansions into Eqs. 19 and 20, the second approximation is obtained by equating coefficients of $\epsilon$ in the equations to zero, giving

$$
\left(\phi_{0} \phi_{g_{1}}\right)^{\prime}+\tau_{g_{1}} / \rho=\alpha_{0} \phi_{0} \frac{d}{d \rho}\left(f_{d} \frac{1}{\tau_{0}^{x}} \phi_{0} \phi_{0}^{\prime} \frac{1}{\xi_{0}^{\prime}}\right)
$$




$$
\tau_{g_{1}}+\frac{\gamma_{0}-1}{\gamma_{0}} \phi_{0} \phi_{g_{1}}=\alpha_{0} \frac{\partial_{0}-1}{\gamma_{0}} f_{\alpha} \frac{1}{\tau_{0}^{x}} \phi_{0}^{2} \phi_{0}^{\prime} \frac{1}{\xi_{0}}+\alpha_{0} \frac{c}{c_{p_{0}}} \beta f_{k} \frac{1}{\tau_{0}^{x}} \phi_{0} \tau_{0}^{\prime} \frac{1}{\xi_{0}^{\prime}}
$$

Eliminating $\tau_{g}$ between these equations

$$
\begin{aligned}
& \left(\phi_{0} \phi_{g_{1}}\right)^{\prime}-\frac{z_{0}-1}{j_{0}} \frac{1}{g}\left(\phi_{0} \phi_{g_{1}}\right) \\
& =\rho^{\frac{y_{0}-1}{\partial_{0}}} \frac{d}{d \rho}\left(\frac{1}{\rho \frac{z_{1-1}}{g_{0}}} \phi_{0} \phi_{g_{1}}\right) \\
& =\tau_{0} \frac{d}{d \rho}\left(\frac{\phi_{0} \phi_{g_{1}}}{\tau_{0}}\right) \\
& =\alpha_{0} \phi_{0} \frac{d}{d g}\left(f_{d} \frac{\phi_{0} \phi_{0}^{\prime}}{\tau_{0}^{n}} \frac{1}{\xi_{0}^{\prime}}\right)-\alpha_{0} \frac{r_{0}-1}{\gamma_{0}} f_{d} \frac{\phi_{0}^{2} \phi_{0}^{\prime}}{\rho \tau_{0}^{n}} \frac{1}{\xi_{0}^{\prime}}-\alpha_{0} \frac{C}{c_{p_{0}}} \beta f_{h} \frac{\phi_{0} \tau_{0}^{\prime}}{\rho \tau_{0}^{n}} \frac{1}{\xi_{0}^{\prime}}
\end{aligned}
$$

and integrating by parts

$$
\frac{\phi_{g_{1}}}{\phi_{0}}=\alpha_{0} f_{d} \frac{\phi_{0}^{\prime}}{\tau_{0}^{x}} \frac{1}{\xi_{0}^{\prime}}-\alpha_{0} \frac{\tau_{0}}{\phi_{0}^{2}} \int_{\rho_{i}}^{\rho}\left(f_{d} \phi_{0}^{\prime 2}+\frac{c}{c_{p_{0}}} \beta f_{h} \frac{\tau_{0}^{\prime}}{\rho}\right) \frac{\phi_{0}}{\tau_{0}^{x+1}} \frac{d \rho}{\xi_{0}^{\prime}}
$$

Substituting this expression for $\phi_{g_{1}} / \phi_{0}$ into Eq. 44

$$
\frac{\tau_{g 1}}{\tau_{0}}=\alpha_{0} \frac{C}{c_{p_{0}}} \beta f_{h} \frac{\phi_{0} \tau_{0}^{\prime}}{\tau_{0}^{k+1}} \frac{1}{\xi_{0}^{\prime}}+\alpha_{0} \frac{\partial_{0}-1}{\gamma_{0}} \int_{\rho_{i}}^{\rho}\left(f_{d} \phi_{0}^{\prime 2}+\frac{C}{\varphi_{p_{0}}} \beta f_{k} \frac{\tau_{0}^{\prime}}{\rho}\right) \frac{\phi_{0}}{\tau_{0}{ }^{n+1} \frac{d \rho}{\xi_{0}^{\prime \prime}}}
$$

It is assumed that the velocity and temperature lags are zero as the flow enters the convergent section of the nozzle where $\rho=\rho_{i}$ and $/ / \xi_{0}^{\prime}=0$. Although here the analysis is restricted to this initial condition, there is no particular difficulty in extending it to any prescribed lags at the entrance to the nozzle provided that care is taken in defining $p_{c}$ correctly. Clearly the pressure is not suitable as an independent variable for ducts of constant cross-sectional area, so calculation of lags in a cylindrical rocket chamber preceding the nozzle must be made in a different way.

It is convenient to introduce new notation to take advantage of similarities in the expressions for $\phi_{g} / \phi_{0}$ and $\tau_{g_{1}} / \tau_{0}$. Let $G(\rho)$ be defined as

$$
G(\rho)=-\alpha_{0}\left(f_{d} \phi_{0}^{\prime 2}+\frac{C}{C_{p_{0}}} \beta f_{k} \frac{\tau_{0}^{\prime}}{\rho}\right) \frac{\phi_{0}}{\tau_{0}^{n+1}}
$$

The function $G(\rho)$ is positive since $\xi_{0}^{\prime}$ is negative for all values of $\rho$, and the remaining terms are positive. With 
substitutions from Eqs. 33-38, $G(S)$ can be expressed as

$$
G(\rho)=\alpha_{0} \Gamma_{0}\left[f_{d} \rho^{\frac{\gamma_{0}-1}{\gamma_{0}}}+2 \frac{c}{c_{p_{0}}} \beta f_{h}\left(1-\rho^{\frac{p_{0}-1}{\gamma_{0}}}\right)\right] \frac{A}{A_{t}} \frac{1}{\rho^{(n+1) \frac{\hbar_{0}-1}{\gamma_{0}}}\left(-\frac{1}{\xi_{0}}\right)}[48]
$$

where the factor $A / A_{t}$ is introduced directly because its magnitude is more immediately evident than is the equivalent form expressed as a function of $\zeta$. Except the factor 1/ $\xi_{0}^{\prime}$, which depends on nozzle shape and will be discussed later, and the factor $A / A_{t}$, the only other variable in the expression for $G$ is $\rho \frac{t_{0} \frac{E_{1}}{\gamma_{0}}}{\gamma_{0}}=\tau_{0}$. This quantity usually will have a small range of variation, from slightly less than unity at the nozzle entrance to 0.5 or so at the nozzle exit.

The fractional contribution of heat transfer to the function $G(P)$ will be represented by $\psi(P)$, where

$$
\psi(\rho)=\frac{2 \frac{C}{c_{p_{0}}} \beta f_{h}\left(1-\rho^{\frac{t_{0}-1}{\gamma_{0}}}\right)}{f_{d} \rho^{\frac{f_{0}-1}{\gamma_{0}}}+2 \frac{C}{c_{p_{0}}} \beta f_{h}\left(1-\rho^{\frac{t_{0}-1}{\gamma_{0}}}\right)}
$$

Introducing the functions $G(S)$ and $\psi(s)$ into the expressions for $\phi_{g_{1}} / \phi_{0}$ and $\Upsilon_{g_{1}} / T_{0}$ given by Eqs. 45 and 46

$$
\begin{aligned}
& \frac{\phi_{g_{1}}}{\phi_{0}}=[1-\psi(\rho)] G(\rho)-\frac{\gamma_{0}-1}{\gamma_{0}} \frac{\rho^{\frac{\gamma_{0}-1}{\gamma_{0}}}}{1-\rho^{\frac{i_{-1}}{\gamma_{0}}}} \int_{\rho_{i}}^{\rho} G(\rho) d(\ln 1 / \rho) \\
& \frac{\tau_{g_{1}}}{\tau_{0}}=-\psi(\rho) G(\rho)+\frac{\gamma_{0}-1}{\gamma_{0}} \int_{\rho_{i}}^{\rho} G(\rho) d(\ln 1 / \rho)
\end{aligned}
$$

The qualitative behavior of the velocity and temperature lags is demonstrated clearly by these equations. Near the nozzle entrance, the first terms dominate since the contributions of the integrals are small. The velocity of the gas increases above the velocity of the mixture with no lag, and the gas temperature drops below the temperature of the mixture with no lag. Further downstream, however, the trends are reversed, as the contribution of the integrals becomes larger. The heat stored in the particles feeds back directly into the gas, and kinetic energy deficiency stored in the particles is transferred to the gas by dissipation. The effects are parallel, and both tend to increase the gas temperature and lower 
the gas velocity. If the heat capacity of the particles is zero, i. e., $C=0$ and hence $\psi=0$, the mechanism of transfer of energy is entirely dissipative, then the velocity lag exhibits the same trends as for finite particle heat capacity, but the gas temperature increment increases monotonically.

\section{EFFECT OF VELOCITY AND TEMPERATURE LAGS ON PERFORMANCE}

The effect of the particle lags on performance will be determined as a correction to the performance of a rocket nozzle of identical shape with the same mass fraction of particles but without lag. The stagnation temperature $T_{c}$ in the rocket chamber is assumed to be the same with and without lags. Expanding the terms in Eq. 18 up to and including the first order in $\epsilon$

$$
\frac{p_{c}}{\min } \frac{A}{\sqrt{R_{0} T_{c}}}=\frac{\tau_{g}}{\rho \phi_{g}} \cong \frac{\tau_{0}}{\rho \phi_{0}}\left[1+\epsilon\left(\frac{\tau_{g 1}}{\tau_{0}}-\frac{\phi_{g}}{\phi_{t}}\right)\right]
$$

Let $\rho_{t_{0}}$ be the throat pressure ratio without lag and $\rho_{t}$ the value with lag; it is assumed that $\left(\rho_{t}-\rho_{t_{0}}\right) / \rho_{t_{0}}$ is of order $\epsilon$. The throat pressure ratio is determined by the condition $A^{i}=0$, hence equating the differential of the logarithm of the right hand side of Eq. 52 to zero and expanding to order $\epsilon$

$$
\frac{\rho_{t}-\rho_{t_{0}}}{\rho_{t_{0}}}=-\epsilon\left[\frac{\tau / \rho \phi}{\rho(\tau / \rho \phi)^{\prime \prime}}\right]_{t_{0}}\left(\frac{\tau_{g 1}}{\tau_{0}}-\frac{\phi_{g 1}}{\phi_{0}}\right)_{t_{0}}^{\prime}
$$

or evaluating the terms in the brackets explicitly

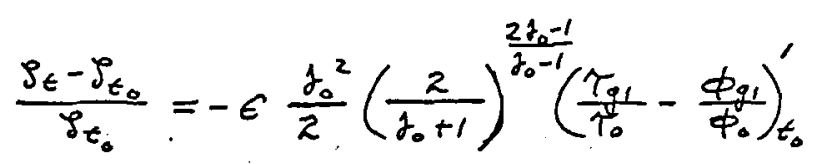

Now $A_{t}$ and $\sqrt{R_{0} T_{c}}$ are the same with and without lag, so an expansion of Eq. 52 around $\rho_{t}=\rho_{t_{0}}$ leads to the relation

$$
\frac{p_{c} / \dot{m}}{p_{c_{0}} / \dot{m}_{0}}=1+\epsilon\left(\frac{\tau_{g l}}{\tau_{0}}-\frac{\phi_{g r}}{\phi_{0}}\right)_{t_{0}}
$$

The throat pressure ratio $\zeta_{t}$ is not needed to find $p_{c} / \dot{m}$ because of the condition $(T / \rho \phi)_{t_{0}}^{\prime}=0$, peculiar to the throat. Substituting from Eqs. 50 and 51 and recalling that

$$
\rho_{t_{0}} \frac{\gamma_{0}-1}{\gamma_{0}}=2 /\left(\gamma_{0}+1\right)
$$




$$
\frac{p_{c} / \dot{m}}{p_{c_{0}} / \dot{m}_{0}}=1-\epsilon\left[G\left(\rho_{\epsilon_{0}}\right)-\int_{\rho_{i}}^{\vartheta_{t_{0}}} G(\rho) d\left(\ln \frac{1}{\rho}\right)\right]
$$

The quantity in the brackets is positive, as will become clear when the behavior of $G(S)$ is examined later; hence $p_{c} / \dot{m}$ always decreases as a result of the lags. Only the ratio $p_{c} / \dot{m}$ is determined in the flow with lag; some additional information must be supplied to determine $p_{c}$ and $\dot{m}$ separately. For a solid propellant rocket this normally would be a burning rate law relating $m$ and $p_{c}$.

The vacuum thrust $F_{v}$ for the nozzle is given by

$$
F_{v}=A_{e}\left[p+(1-\alpha) \rho u_{g}^{2}+\alpha \rho u_{s}^{2}\right]_{e}
$$

where the subscript $e$ denotes the nozzle exit. The vacuum specific impulse is defined by

$$
I_{v}=F_{v} / \dot{m} g
$$

These expressions are exact, within the limitations of onedimensional flow, as is the derived form

$$
I_{v}=A_{e} \frac{p_{c}}{i m} \rho_{e}\left[1+\frac{\phi_{g}^{2}}{\tau_{g}^{2}}-\alpha_{0} \frac{\phi_{g}}{\tau_{g}}\left(\phi_{g}-\phi_{s}\right)\right]_{e}
$$
Substituting the expansions for $\phi_{g}, \tau_{g}$, and $\phi_{s}$ and re-
taining terms of order $\in$

$$
\left.I_{v} \cong A_{e} \frac{\phi_{c}}{\dot{i}_{i}} \rho_{e}\left[1+\left(\frac{\phi_{0}^{2}}{T_{c}}\right)_{e}+\epsilon\left\{\frac{\phi_{0}^{2}}{\tau_{0}}\left(2 \frac{\phi_{g}}{\phi_{0}}-\frac{T_{g}}{T_{0}}\right)-\alpha_{0} \frac{\phi_{0}}{T_{0}}\left(\phi_{g}-\phi_{s}\right)\right\}\right\}_{e}\right][56]
$$

The exit pressure can be determined from the continuity equation

$$
\frac{p_{c}}{\dot{m}} \frac{A_{e}}{\sqrt{\pi_{0} T_{c}}}=\left(\frac{\tau_{g}}{\rho \phi_{g}}\right)_{e} \bumpeq\left(\frac{\tau_{0}}{\rho \phi_{0}}\right)_{e}+\epsilon\left[\frac{\tau_{0}}{\rho \phi_{0}}\left(\frac{\tau_{g l}}{\tau_{0}}-\frac{\phi_{g r}}{\phi_{0}}\right)\right]_{e_{0}}
$$

Expanding the expression for $\left(\tau_{0} / \rho \phi_{0}\right)_{e}$ to the first power in $\left(\rho_{e}-\rho_{e_{0}}\right) / \rho_{e_{0}}$, which is assumed to be of order $\epsilon$, and substituting for $p_{0} / \dot{m}$ from Eq. 54

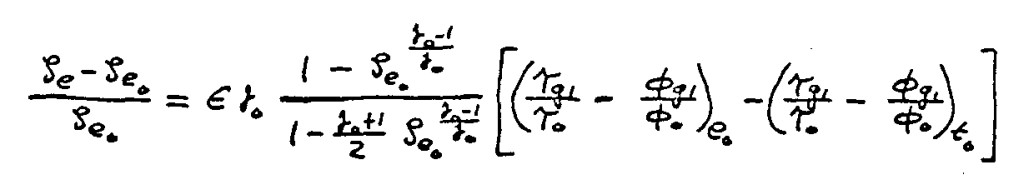


The quantity $\rho_{e}\left[1+\phi_{0}^{2} / \tau_{0}\right]_{e}$ in the expression for $I_{v}$, Eq. 56 , is expanded to the first power in $\left(\rho_{e}-s_{e_{0}}\right) / \rho_{e_{c}}$, and with substitutions from Eqs. 41,50 , and 51 the specific impulse can be expressed as

$$
\frac{I_{v}}{I_{v_{0}}}=1-\epsilon \frac{\frac{\gamma_{0}-1}{2 \gamma_{0}} \rho_{e_{0}}^{\frac{\gamma_{0}-1}{\gamma_{0}}}}{1-\frac{\partial_{0}+1}{2 \gamma_{0}} \rho_{e_{0}}^{\frac{\delta_{0}-1}{\gamma_{0}}}}\left[G\left(\rho_{t_{0}}\right)+\int_{\rho_{t_{0}}}^{\rho_{e_{0}}} G(\rho) d\left(\ln \frac{1}{\rho}\right)\right]
$$

This formula shows that the vacuum specific impulse is quite independent of conditions upstream of the throat, a result that does not appear obvious.

The thrust can be determined only if $\beta_{c}$ or $\dot{m}$ or a relation between them is prescribed. For instance, in a solid propellant rocket with $\dot{m} / \dot{m}_{0}=\left(p_{c} / p_{c_{0}}\right)^{N}$

$$
\frac{F_{v}}{F_{v_{0}}}=\frac{I_{v}}{I_{v_{0}}}-\frac{1-N}{N}\left(1-\frac{p_{c} / \dot{m}}{p_{c_{0}} / \dot{m}_{0}}\right)
$$

where $I_{v} / I_{v_{0}}$ and $\left(p_{c} / \dot{m}\right) /\left(\phi_{c_{0}} / \dot{m}_{0}\right)$ are given by Eqs. 55 and 58 respectively. The percentage reduction of thrust is always greater than the percentage reduction of specific impulse as a result of velocity and temperature lags.

NOZZLE SHAPE PARAMETER $\xi_{0}^{\prime}$

The nozzle shape appears in the analysis through the parameter $\xi$ only. This parameter occurs directly as a factor in the function $G(\rho)$ defined by Eq. 48 and indirectly, with weaker effect, through the coefficients $f_{\alpha}$ and $f_{h}$, also occurring in $G(S)$. If $\theta$ is the angle between the tangent to the nozzle contour and the nozzle axis, with $\theta$ positive downstream of the throat

$$
\tan \theta=\frac{d x}{d x}=\frac{d\left(x / r_{t}\right)}{d \xi_{0}}=\frac{d\left(A / A_{t}\right)^{1 / 2}}{d \rho} \frac{d s}{d \xi_{0}}
$$

and hence

$$
-\frac{1}{\xi_{0}^{\prime}}=-\frac{d \rho}{d \xi_{0}}=-2 \sqrt{\frac{A_{t}}{A}} \frac{\tan \theta}{A^{\prime} / A}
$$

or, substituting from Eq. 35

$$
-\frac{d \rho}{d \xi}=2 \xi_{0} \sqrt{\frac{A_{t}}{A}} \rho\left(1-\rho^{\frac{\partial_{0}-1}{\gamma_{0}}}\right) \frac{\frac{t}{t_{0} \theta}}{1-\rho^{\frac{\partial_{0}-1}{\gamma_{0}}}}
$$


where $\rho_{t_{0}}=\left[2 /\left(f_{0}+1\right)\right]^{\frac{t_{0}}{\gamma_{0}-1}}$ is the throat pressure ratio. Proceeding downstream from the throat, the quantity

$$
\left(1-\rho^{\frac{t_{0}-1}{r_{0}}}\right) /\left\{1-\left(\rho / \rho_{t_{0}}\right)^{\frac{t_{0}-1}{t_{0}}}\right\}
$$

decreases as do the factors $\sqrt{A_{t} / A}$ and $\rho$, the latter very rapidly. Hence $-d \rho / \alpha \xi$ decreases rapidly after $\theta$ reaches its maximum value downstream of the throat. The trend is more marked for a bell-shaped nozzle ( $\theta$ decreasing) than for a conical nozzle ( $\theta=$ constant).

The value of $-d s / d \xi$ at the throat is indeterminate as given by Eq. 60. An expansion in powers of $\left(\rho_{t}-\rho_{t_{0}}\right) / \rho_{t_{0}}$ near the throat yields the relation

$$
-\frac{d \rho}{d \xi_{0}} \rightarrow \frac{1}{\sqrt{b_{t}}} f_{0}\left(\frac{2}{g_{0}+1}\right)^{\frac{3 \gamma_{0}-1}{2\left(r_{0}-1\right)}}\left(1-\frac{3-2 \gamma_{0}}{3 f_{0}} \cdot \frac{\rho-\rho_{t_{0}}}{\rho_{t_{0}}}+\cdots\right)[6 I]
$$

where $b_{t} r_{t}$ is the radius of curvature of the nozzle contour at the throat. Since $-d \rho / d \xi_{0}$ is increasing downstream at the throat, the maximum value of $-d \rho / d \xi_{0}$ must occur somewhere downstream of the throat.

If the nozzle entrance joins a cylindrical rocket chamber with a continuous slope and a discontinuity in curvature, the behavior of $-d \rho / d \xi_{0}$ near the entrance can be determined by an expansion that gives

$$
-\frac{d \rho}{d \xi_{0}} \rightarrow \frac{1}{\sqrt{b_{i}}}\left(\frac{A_{t}}{A_{i}}\right)^{5 / 4} \sqrt{\frac{\gamma_{0}-1}{\gamma_{0}}}\left(\frac{2}{\gamma_{0}+1}\right)^{\frac{3 \gamma_{0}-1}{2\left(\gamma_{i}-1\right)} \sqrt{\left(\rho_{i}-\rho\right) / \rho_{i}}} \frac{1-\left(\rho_{t_{0}}\left(\rho_{i}\right)^{\frac{\gamma_{0}-1}{\gamma_{0}}}\right.}{1}
$$

where $A_{i}$ is the cross-sectional area of the nozzle entrance and $b_{i} r_{t}$ is the radius of curvature of the nozzle contour immediately downstream of the entrance. This relation shows that $-d \varphi / d \xi_{0}$ remains finite but has an infinite slope at the nozzle entrance.

The general behavior of $-d \rho / d \xi$ os a function of $\ln \frac{1}{5}$, say, can be deduced from Eqs. 60, 61, and 62. For a typical nozzle, the value of $-d \& / d \xi$ increases sharply from zero at the nozzle entrance, then rather slowly to a maximum downstream of the throat, and decreases to a small quantity at the nozzle exit. The maximum is quite close to the value at the throat; hence the contour radius at the throat is the primary influence on the magnitude of $-d \rho / d \xi$.

A discontinuity in radius of curvature at the throat 
produces a discontinuity in $-\alpha \rho / \alpha \frac{3}{0}$. Elsewhere on the nozzle contour a discontinuity in curvature with continuous slope produces only a discontinuity in the derivative of $-d \rho / d \xi_{0}$. It is unrealistic, in a one-dimensional approximation, to prescribe a nozzle contour that produces discontinuities in $-d \rho / d \xi_{0} ;$ such discontinuities would be smoothed out by the real three-dimensional flow.

\section{DRAG AND HEAT TRANSFER COEFEICIENTS $f_{\alpha}$ AND $f_{h}$}

The drag and heat transfer coefficients introduced in Eqs. 6 and 7 to allow for departures from Stokes' regime for drag and simple conduction for heat transfer are the most difficult parameters to evaluate in the analysis. Below a Reynolds number of unity, for the Mach number sufficiently low, the Stokes' drag formula and conduction theory $\left(f_{d}=f_{k}=1\right)$ are adequate. However, this regime of relative flow is applicable only to very small particles and leads to negligible lags in most examples of practical interest. The magnitude of the drag coefficient of spheres at very low Mach number has been well established by measurement over a wide range of Reynolds numbers and hence can be evaluated from the relation $f_{d}=24 /\left(C_{D} R_{e}\right)$, where $C_{D}\left(=2 D / \pi a^{2} \rho u^{2}\right)$ is the measured drag coefficient and $R_{e}$ is the Reynolds number based on sphere diameter. The heat transfer coefficient for spheres, usually presented as the Nusselt number $N_{k}\left(=2 / f_{h}\right)$, has also been measured over a wide range of Reynolds numbers at low Mach number, although the magnitude is not quite as well established as that of the drag coefficient.

These remarks apply to continuum flow, which occurs only when the ratio of the mean free path of the molecules is very small compared with the particle dimension. The criterion for continuum flow is that the value of $M / T_{e}$, where $M$ is the Mach number of the flow relative to the particle, should be very small compared with unity. This condition is not satisfied for typical particles in a rocket nozzle; hence dependence of $f_{d}$ and $f_{h}$ on $M / R_{e}$ must be investigated.

Measurements of the heat transfer to spheres in the slip flow regime have been made by Kavanau (5). His measurements covered the Mach number range from 0.1 to 0.7 and the Reynolds number range from 2 to 100. To represent the results of the experiments in a simple form, Kavanau pro-

1 Numbers in parenthesis indicate References at end of paper. 
posed a semi-empirical interpolation formula, which can be written in the present notation as

$$
f_{h}=f_{h}^{(0)}+K \frac{M}{\pi_{e} P_{r}}
$$

where $f_{h}^{(0)}\left(=2 / N_{k}^{(0)}\right)$ is the value of $f_{h}$ for continuum flow $(M=0)$ and $K$ is a constant; Kavanau found that $K=6.84$ gave the best agreement with his measurements. The second term on the right-hand side was suggested by free molecular flow theory, with the reasonable assumption that if the formula gives the correct value of $f_{h}$ in continuum flow when the second term is zero and in free molecular flow when the second term is dominant, the formula may well give a good approximation in the slip and transition regimes between the two extremes.

If the expression for heat transfer to a sphere in free molecular flow (e.g., Ref. 6) is expanded in a power series in $M^{2}$, the leading terms for $f_{h}=2 / N_{u}$ are given by

$$
f_{h}=\frac{2 \sqrt{2 \pi}}{\bar{\alpha}} \frac{j^{3 / 2}}{j+1} \frac{M}{\operatorname{R}_{e} T_{r}}\left(1-\frac{j}{6} M^{2}+\cdots\right)
$$

where $\bar{\alpha}$ is the accommodation coefficient. Taking $f=1.4$ to compare with Kavanau's experiments in air and assuming that $\bar{\alpha}=1.0$, the numerical factor in Eq. 63 is 6.92 , very close to the value 6.84 that was determined experimentally. Kavanau used the results of an approximate theory of Sauer (7) to evaluate $f_{k}^{(0)}$, and this agreed well with his experiments at the lowest Mach number. The analytical expression for $f_{k}^{(a)}$ is complex, but it can be represented reasonably well by an empirical relation

$$
\begin{aligned}
\log _{10} \frac{1}{f_{h}^{(0)}} & =0.108\left(\log _{10} T_{e}+0.5\right)^{1.86} & & R_{e}>10^{-0.5} \\
& =0 & & \pi_{e}<10^{-0.5}
\end{aligned}
$$

where the dependence on Prandtl number is ignored. For application to a rocket nozzle, a value of $\mathrm{g}=1.28$ in Eq. 63 seems more appropriate; hence it will be assumed that the heat transfer correction factor is

$$
f_{h}=f_{h}^{(0)}+6.35 \frac{M}{R_{e} P_{r}}
$$

with $f_{h}^{(0)}$ given by Eq. 64. This formula is expected to be sufficiently accurate as long as $M$ is not much larger than unity.

The drag of spheres apparently has not been measured in the slip flow regime at subsonic Mach numbers. It seems reasonable, however, to use the same procedure for 
estimating drag as for heat transfer. Expanding the expression for drag in terms of $f_{d}$ for the regime of free molecular flow in powers of $M^{2}$, it is found that, with reflection coefficients equal to unity and with the ratio of gas temperature to sphere temperature close to unity

$$
f_{d}=\frac{18 \sqrt{2 \pi}}{\pi+8} \sqrt{2} \frac{M}{R_{e}}\left[1-\frac{4 r}{\delta(\pi+8)} M^{2}+\cdots\right]
$$

For $\gamma=1.28$, the numerical factor of $M / R_{e}$ is 4.58 , so it is assumed that

$$
f_{\alpha}=f_{\alpha}^{(0)}+4.58 \frac{M}{T_{e}}
$$

where $f_{d}^{(0)}$ is the value of $f_{d}$ for continuum flow. This latter coefficient has been measured by several independent investigators (e. g., Ref. 8), and the results can be approximated for Reynolds numbers up to $10^{4}$ by the empirical formula

$$
\begin{aligned}
\log _{10} \frac{1}{f_{d}^{(0)}} & =0.1025\left(\log _{10} \pi_{e}+0.5\right)^{2} & & R_{e}>10^{-0.5} \\
& =0 & & R_{e}<10^{-0.5}[67]
\end{aligned}
$$

The combination of Eqs. 66 and 67 should give, by analogy with the heat transfer findings, a satisfactory representation of $f_{d}$ as a function of $\pi_{e}$ and $M / \pi_{e}$, at least for $M<1$.

The parameters $\mathcal{R}_{e}$ and $M / \mathcal{R}_{e}$, which determine the coefficients $f_{d}$ and $f_{h}$, must be evaluated first. The ratio $M / \pi_{e}$ is, by definition

$$
\frac{M}{T_{e}}=\frac{\mu}{\rho_{g} \cdot 2 a \cdot \sqrt{r R T_{g}}}
$$

Substituting from Eqs. 13, 21, and 33 and keeping only the first approximation

$$
\frac{M}{R_{e}}=\frac{\delta}{\sqrt{1-\alpha_{0}} \sqrt{j}}\left(\frac{1}{\rho}\right)^{1-\left(n+\frac{1}{2}\right) \frac{\partial_{0}-1}{\delta_{0}}}
$$

where $\delta$ is a new dimensionless parameter defined by

$$
\delta=\frac{\mu_{c} \sqrt{T_{0} T_{c}}}{2 a p_{c}}
$$

The Reynolds number $T_{e}$ is defined by the equation

$$
T_{e}=\left(u_{g}-u_{s}\right) z a \rho_{g} / \mu
$$

Substituting from Eqs. 13, 21, 33, 34, and 41 and retaining only the first approximation 
DETONATION AND TWO-PHASE FLOW

$$
\frac{T_{e}}{f_{\alpha}}=\frac{\epsilon}{\delta}\left(1-\alpha_{0}\right)\left(\frac{1}{\rho}\right)^{2 n \frac{\gamma_{0}-1}{t_{0}}}\left(-\frac{1}{\xi_{0}^{\prime}}\right)
$$

Because the combination $T_{e} / f_{d}$ rather than $T_{e}$ alone is determined by the particle and nozzle characteristics, it is convenient to have $f_{d}$ as a function of $R_{e} / f_{d}$ and $M / R_{e}$ so that $f_{d}$ can be found directly without iteration. From Eqs. 66 and 67 it is a simple matter to plot $f_{d}$ vs. $R_{e} / f_{d}$ with $M / R_{e}$ as a parameter, as is shown in Fig. 1. The line corresponding to $M=1$ is shown in the figure; values of $f_{d}$ for points falling to the right of this line may not be reliable. After $f_{d}$ is read from the chart, the value of $R_{e}$ can be found and $f_{h}$ calculated directly from Eqs. 64 and 65.

The value of $\operatorname{Re} / f_{d}$ for $\epsilon$ and $\delta$ fixed depends on a power of the pressure ratio that has limited variation and on the shape parameter $\xi_{0}^{\prime}$. From the discussion in the previous section it is apparent that $R_{e} / f_{d}$ will have a maximum somewhat downstream of the nozzle throat. The value of $M / R_{e}$, on the other hand, is independent of the nozzle shape and increases monotonically from entrance to exit; hence the effects of slip flow are most pronounced near the exit. Since $M / R_{e}$ is inversely proportional to particle radius, the factors $f_{d}$ and $f_{k}$ can become large for sufficiently small particles. However, the lags are proportional to the combinations $\epsilon f_{d}$ and $\epsilon f_{h}$, which become small as the particle radius is reduced.

Example

The large number of parameters entering the calculation of particle lags makes a general discussion difficult, so an examination of one or more specific examples is almost essential as a starting point. Calculations have been carried out for the following arbitrary, but it is hoped reasonable, choice of characteristics.

$$
\begin{array}{lll}
c_{p}=0.500 \mathrm{Btu} / 16-{ }^{\circ} \mathrm{F} & p_{c}=1000 \mathrm{psi} & a=2.5 \times 10^{-4} \mathrm{~mm} \\
R=0.110 \mathrm{Btu} / 16-{ }^{\circ} \mathrm{F} & T_{c}=6000^{\circ} \mathrm{R} & \alpha_{0}=0.40 \\
C=0.550 \mathrm{Btu} / 76-{ }^{\circ} \mathrm{F} & \mu_{c}=1.5 \times 10^{-6} \mathrm{76}-\mathrm{sec} / \mathrm{ft} .^{2} & P_{r}=0.74 \\
\rho_{s}=210 \mathrm{lb} / \mathrm{ft}^{3} & r_{t}=3.00 \mathrm{in} . &
\end{array}
$$

Dimensionless parameters required in the analysis are then 
DETONATION AND TWO-PHASE FLOW

$$
\begin{array}{lll}
\gamma=1.282 & \epsilon=0.817 & \delta=2.00 \times 10^{-3} \\
\gamma_{0}=1.1454 & c / c_{p_{0}}=1.058 & \beta=1.222
\end{array}
$$

The nozzle contour chosen for the example is shown in Fig. 2. The nozzle is made up of three sections, as follows:

$$
\begin{array}{rlrl}
\text { (i) } r / r_{t} & =1.468-(1-\cos \theta) & 0 \leqslant 1 \theta \mid \leqslant 40^{\circ} \\
\text { (ii) } r / r_{t} & =1+(1-\cos \theta) & -40^{\circ} \leqslant \theta \leqslant 28^{\circ} \\
\text { (iii) } r / r_{t}=3.438-8.21 \tan ^{2} \theta & 28^{\circ} \geqslant \theta \geqslant 13^{\circ}
\end{array}
$$

The first two sections are circular $\operatorname{arcs}\left(b_{i}=b_{t}=1\right)$ and the third is a two-parameter curve. For this contour $A_{i} / A_{t}=2.155$ and $A_{e} / A_{t}=9.0$; the length of the convergent part is $1.29 r_{t}$, and the length of the divergent part is $5.42 r_{t}$. Without lag, the vacuum thrust and specific impulse would be $50,000 \mathrm{Ib}$ and $271 \mathrm{sec}$ respectively, with constant thermodynamic properties, and the exit pressure ratio $p_{c} / p_{e_{0}}=59.6$.

A graph of the shape parameter $-d \rho / d \xi$ plotted against $\ln (1 / 9)$ is shown in Fig. 3. The kinks occur at the two points where the radius of curvature of the nozzle contour changes. In the same figure, the function defined by Eq. 48 is shown. The value of the Reynolds number rises to 56 immediately downstream of the throat and decreases to 2 at the nozzle exit. The coefficient $f_{d}$ drops to 0.32 at the throat and increases to 1.22 at the exit, whereas $f_{h}$ has a minimum of 0.36 near the throat and rises to 1.52 at the exit.

The functions $\epsilon \phi_{g_{1}} / \phi_{0}, \epsilon \phi_{s_{1}} / \phi_{0}, \epsilon \tau_{g_{1}} / \tau_{0}$, and $\epsilon \tau_{s_{1}} / \tau_{0}$ are plotted against $\ln (1 / \rho)$ in Fig. $4_{0}$. The criterion for validity of the analysis is that these quantities should be small compared with unity, a condition that is fairly well satisfied in this particular example. Appreciably larger diameters of the particles would give less reliable results. For instance, doubling the particle radius to $5.0 \times 10^{-4} \mathrm{~cm}$ increases the value of $\pi_{e} / f_{d}$ by a factor of 8 , and this, in the throat region, reduces $f_{d}$ and $f_{h}$ by a factor of 2 or so. Since the lags are linear in $\epsilon f_{d}$ and $\epsilon f_{h}$, the values of the perturbation quantities above are doubled in the neighborhood of the throat.

The quantities that are required for performance estimation are listed below with their numerical values. 


$$
\begin{aligned}
& G\left(\rho_{t_{0}}\right)=0.1605 \\
& G\left(\rho_{e_{0}}\right)=0.0994
\end{aligned}
$$$$
\begin{aligned}
& \int_{\rho_{i_{i}}}^{\rho_{t_{\theta}}} G(\rho) d\left(\ln \frac{1}{\rho}\right)=0.0680 \\
& \int_{\rho_{\Phi_{\theta_{0}}}}^{\rho_{e_{0}}} G(\rho) d\left(\ln \frac{1}{\rho}\right)=0.6303
\end{aligned}
$$

Substituting these into Eqs. 55 and 58

$$
\frac{p_{0} / p_{c_{0}}}{\dot{m} / \dot{m}_{0}}=1-0.0753 \quad I_{\sigma} / I_{v_{0}}=1-0.0538
$$

so that the lags are responsible for a reduction of 5.4 percent in specific impulse in this particular example.

\section{DISTRIBUTED PARTICLE SIZE}

The particles in the rocket chamber apparently are formed in a variety of sizes rather than a single size, to which the analysis so far has been restricted. Let $\lambda(a) d a$ be the weight fraction of the total mixture (including gas) which is in the form of particles with radii between $a$ and $a+d a$. Then

$$
\int_{0}^{\infty} \lambda(a) d a=\alpha \quad \int_{0}^{\infty} \lambda_{0}(a) d a=\alpha_{0}
$$

where $\alpha$ is the total weight fraction of particles and the subscript 0 refers to no lag. Then Eq. 1 is still valid, as is the continuity equation for the gas, Eq. 2. The particle velocity $u_{s}$ is now a function of $a$; hence the continuity equation for particles of radius between $a$ and $a+d a$ is

$$
\lambda \text { (a) } \rho u_{s}(a) A=\lambda_{0} \text { (a) } \dot{m}
$$

which replaces Eq. 3. Dividing Eq. 73 by Eq. 2 gives

$$
\lambda u_{s}=\frac{1-\alpha}{1-\alpha_{0}} \lambda_{0} u_{g}
$$

which is the counterpart of Eq. 9.

The momentum equation for the mixture is now

$$
(1-\alpha) \rho u_{g} \frac{d u_{g}}{d x}+\rho \int_{0}^{\infty} \lambda u_{s} \frac{d u_{s}}{d x} d a+\frac{d p}{d x}=0
$$


the extension of Eq. 4. Substituting for $\lambda u_{s}$ from Eq. 74 and replacing $(1-\alpha) \rho$ by $\rho_{g}$, the momentum equation becomes

$$
u_{g} \frac{d u_{g}}{d x}-u_{g} \int_{0}^{\infty} \lambda_{0}\left(\frac{d u_{g}}{d x}-\frac{d u_{s}}{d x}\right) d a+\frac{1-\alpha_{0}}{\rho_{g}} \frac{d p}{d x}=0
$$

or, in terms of the dimensionless variables, to compare with Eq. 19

$$
\phi_{g} \frac{d \phi_{g}}{d \rho}+\frac{\tau_{g}}{\rho}=\phi_{g} \int_{0}^{\infty} \lambda_{0}\left(\frac{d \phi_{g}}{d \rho}-\frac{d \phi_{g}}{d \rho}\right) d a
$$

The energy equation can be extended in the same way to give

$$
\tau_{g}+\frac{1}{2} \frac{\gamma_{0}-1}{\gamma_{0}} \phi_{g}^{2}=1+\frac{\gamma_{0}-1}{2 \gamma_{0}} \int_{0}^{\infty} \lambda_{0}\left(\phi_{g}^{2}-\phi_{s}^{2}\right) d a+\frac{c^{\prime}}{c_{p_{0}}} \int_{0}^{\infty} \lambda_{0}\left(\tau_{g}-\tau_{s}\right) d a[76]
$$

which replaces Eq. 20; Eq. 18 of course does not change. The relations for drag and heat transfer, Eqs. 26 and 27, were derived for single particles and hence are still valid. All of these equations are exact, within the limitations of the one-dimensional flow approximation.

The first approximation to the solution of the new equations is the same as before because no lags are involved. The parameter $\epsilon$ depends on the particle radius $a$, so it is not suitable as an expansion parameter; it can be replaced by $\epsilon_{m}$ where

$$
\epsilon_{m}=\frac{2}{q} a_{m}^{2} \frac{\rho_{s} \sqrt{T_{0} T_{c}}}{r_{t} \mu_{c}}
$$

and $a_{m}$ is some arbitrary mean particle radius used as a reference. Then

$$
\begin{aligned}
& \phi_{g}=\phi_{0}+\epsilon_{m} \phi_{g_{1}}+\cdots \\
& \tau_{g}=\tau_{0}+\epsilon_{m} \tau_{g 1}+\cdots
\end{aligned}
$$

but in Eqs. 26 and 27 the $\epsilon$ is replaced by $\epsilon_{m}\left(a / a_{m}\right)^{2}$, so that Eqs. 41 and 42 become

$$
\begin{aligned}
& \phi_{g_{1}}-\phi_{s_{1}}=\left(\frac{a}{a_{m}}\right)^{2} f_{d} \frac{\phi_{0} \phi_{0}^{\prime}}{\tau_{0}^{n}} \frac{1}{\xi_{j}^{\prime}} \\
& \tau_{g_{1}}-\tau_{s_{1}}=\left(\frac{a}{a_{n 1}}\right)^{2} f_{h} \beta \frac{\phi_{0} \tau_{0}^{\prime}}{\tau_{0}^{n}} \frac{1}{\xi_{0}^{\prime}}
\end{aligned}
$$


Substituting the expansions into Eqs. 75 and 76 and picking out the coefficients of $\epsilon_{m}$, it is seen that $\phi_{s_{1}}$ and $\tau_{s_{1}}$ appear only in the combinations

$$
\int_{0}^{\infty} \lambda_{0}\left(\phi_{g_{1}}-\phi_{s_{1}}\right) d a \quad \int_{0}^{\infty} \lambda_{0}\left(\tau_{g_{1}}-\tau_{s_{1}}\right) d a
$$

In Eqs. 78 and 79 only the first two factors on the right-hand sides depend on $a$. Defining two new coefficients $F_{d}$ and

$F_{h}$ by the equations

$$
F_{d}=\int_{0}^{\infty} \lambda_{0}\left(\frac{a}{a_{m}}\right)^{2} f_{d} d a \quad F_{h}=\int_{0}^{\infty} \lambda_{0}\left(\frac{a}{a_{n}}\right)^{2} f_{h} d a
$$

it is seen that it is merely necessary to replace $f_{d}$ by $F_{d}$, $f_{h}$ by $F_{h}$, and $\epsilon$ by $\epsilon_{m}$ to make the entire analysis applicable to flow with particles of any distribution of size.

Eqs. 80 can be interpreted in terms of a single particle radius that is equivalent in effect to the distribution at each axial station. For the Stokes' regime of flow the result is very simple, because $f_{d}=f_{h}=1$; then the effective radius is constant through the nozzle and is determined by the second moment of the particle weight distribution function. Outside of the Stokes' ${ }^{\prime}$ regime, however, the equivalent single particle radius is not constant through the nozzle. It has been seen that $f_{d}$ and $f_{h}$ decrease with increasing $a$ in the neighborhood of the throat where the Reynolds number is high. Hence the equivalent single particle radius corresponds to a moment of some power less than 2 in the throat region. Near the nozzle exit slip flow phenomena can become important, and, because $f_{d}$ and $f_{h}$ increase with decreasing size of the particles, the equivalent single particle radius is again less than that determined from the second moment.

The numerical evaluation of $F_{d}$ and $F_{h}$ is straightforward. At each value of $\ln (1 / 9)$ chosen for computation, $M / R_{e}$ and $R_{e} / f_{d}$ are determined from Eqs. 68 and 70 as before, but for several different values of $a$. Then $f_{d}$ and $f_{h}$ are found for each of the values of $a$ and the integrations indicated in Eq. 80 are performed. After $F_{d}$ and $F_{k}$ have been determined as functions of $\ln (1 / 9)$, the method of calculation is the same as for uniform particle radii.

\section{CONCLUDING REMARKS}

The perturbation analysis of particle lags in a rocket nozzle has been shown to lead to reasonably simple expressions for the specific impulse and other performance characteristics of the nozzle for sufficiently small particles. The expansion parameter $\epsilon$ (or $\epsilon_{m}$ for distributed particle size) is proportional to the square of the particle radius and 


\section{DETONATION AND TWO-PHASE FLOW}

inversely proportional to the radius of the nozzle throat. Because the flow relative to the particles is outside the Stokes' regime in practice, the lags are not as strongly dependent on particle radius and throat radius as the proportionality with $\epsilon$ would indicate. The influence of the nozzle shape on particle lags has been shown to depend primarily on the ratio of the radius of curvature of the nozzle contour at the throat to the throat radius. The lags are inversely proportional to the square root of this ratio in the region of the throat, although again, through the effect of deviations from Stokes' regime of flow, the influence is weaker than direct proportionality with the inverse square root of the radius ratio would indicate.

Recent measurements of particle size distributions in rocket nozzles made by Sehgal (9) indicate that the practical range of particle size lies within the scope of the perturbation analysis if the rocket delivers more than 10,000 to $15,000 \mathrm{Ib}$ thrust. For instance, Sehgal reports that 50 percent of the mass fraction of particles is contained in the particles with radii between $1.35 \times 10^{-4} \mathrm{~cm}$ and $2.05 \times 10^{-4} \mathrm{~cm}$ at $500 \mathrm{psi}$ chamber pressure. If the effective single particle radius is about $1.7 \times 10^{-4} \mathrm{~cm}$, the value of $\epsilon$ in the example is the same if the throat radius is reduced from 3 in. to 1.5 in., i. e., the thrust is reduced to $12,500 \mathrm{lb}$. Geometrically similar rockets of larger size would have smaller lags and the accuracy of the perturbation analysis would improve correspondingly.

\section{REFERENCES}

1 M. Gilbert, L. Davis, and D. Altman: Velocity Lag of Particles in Linearly Accelerated Combustion Gases. Jet Propulsion, January 1955, vol. 25, pp. 25-30.

2 M. Barrère, A. Jaumotte, B. F. de Veubeke, and J. Vandenkerchove: Rocket Propulsion, p. 103, Elsevier, Amsterdam, 1960.

3 J. R. Kliegel: One-Dimensional Flow of a GasParticle System. IAS Paper No. 60-3, presented at the 28th annual meeting of the IAS, New York, January 1960.

4 W. S. Bailey, E. N. Nilson, R. A. Serra, and T. F. Zupnik: Gas Particle Flow in an Axisymmetric Nozzle. ARS Jour., June 1961, vol. 31, pp. 793-798.

5 L. L. Kavanau: Heat Transfer from Spheres to a Rarefied Gas in Subsonic Flow. Trans. ASME, July 1955, vol. 77, pp. 617-623. 
6 S. A. Schaaf and L. Talbot: Handbook of Supersonic Aerodynamics, Section 16, Mechanics of Rarefied Gases, Superintendent of Documents, U. S. Government Printing Office, February 1959.

7 R. M. Drake, F. M. Sauer, and S. A. Schaaf: Forced Convection Heat Transfer from Cylinders and Spheres in a Rarefied Gas Flow. Rept. HE-150-74, Univ. of Calif. Engineering Projects, November 1950.

8 S. Goldstein (ed. ): Modern Developments in Fluid Dynamics, Vol. II, pp. 492-493, Oxford University Press, Oxford, 1938 .

9 R. Sehgal: Personal communication. Jet Propulsion Laboratory, Pasadena, Calif., January 30, 1962. 
DETONATION AND TWO.PHASE FLOW

\section{FIGURE CAPTIONS}

Fig. 1 Drag correction factor $f_{\alpha}$

Fig. 2 Nozzle contour

Fig. 3 Shape parameter $-d \rho / d \xi$ and function $G(\rho)$

Fig. 4 Perturbation velocities and temperatures for gas and particles 


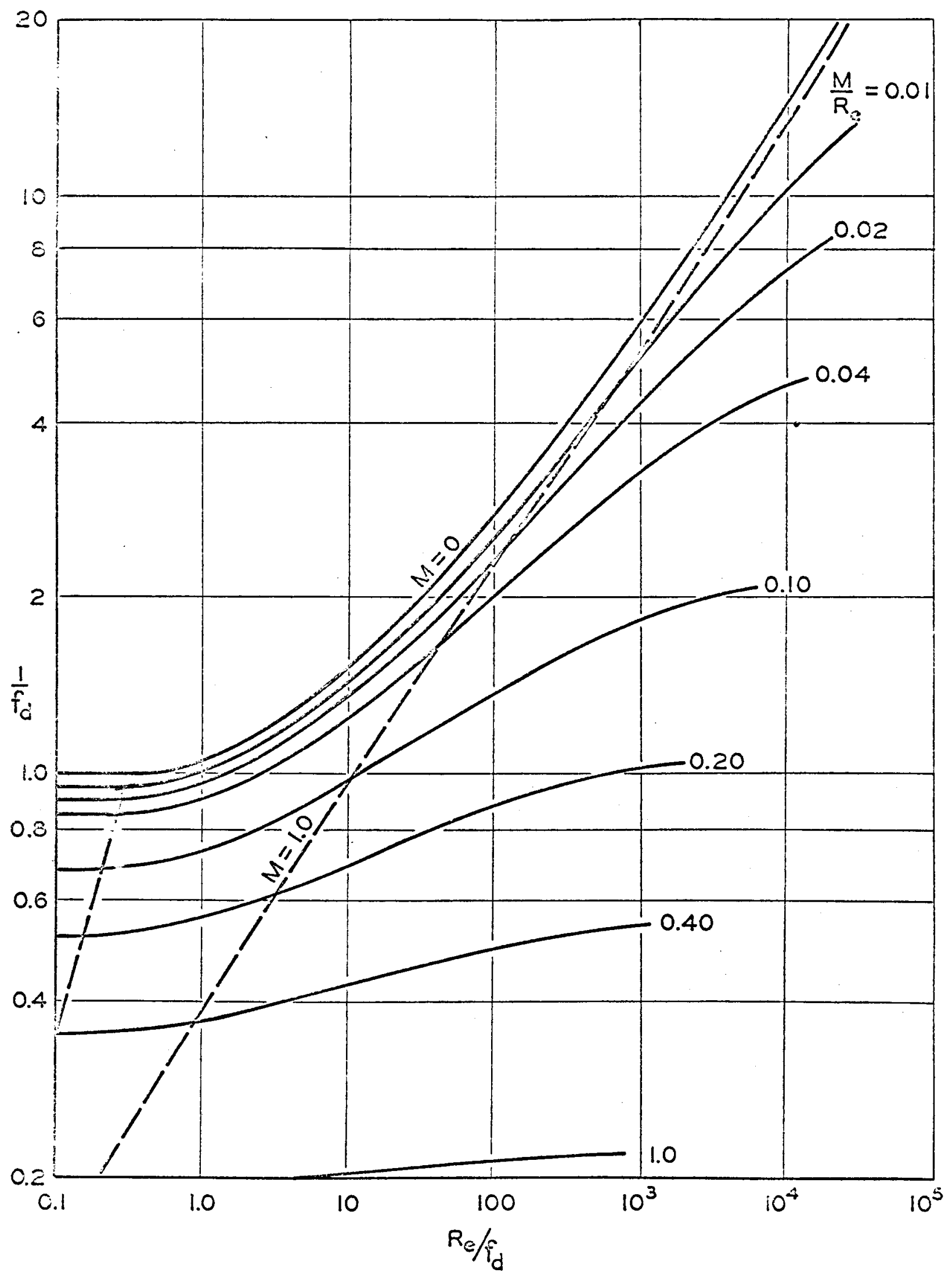


2

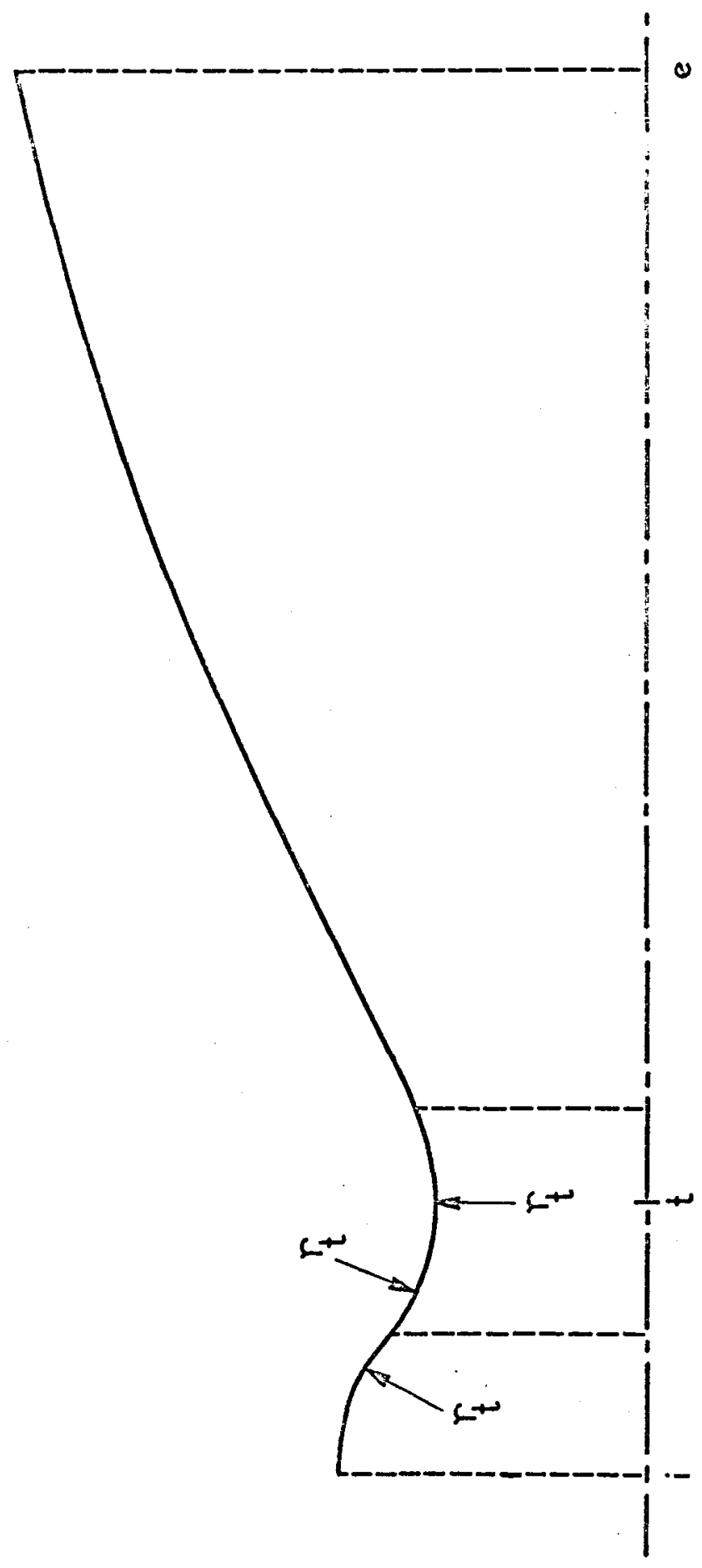


3

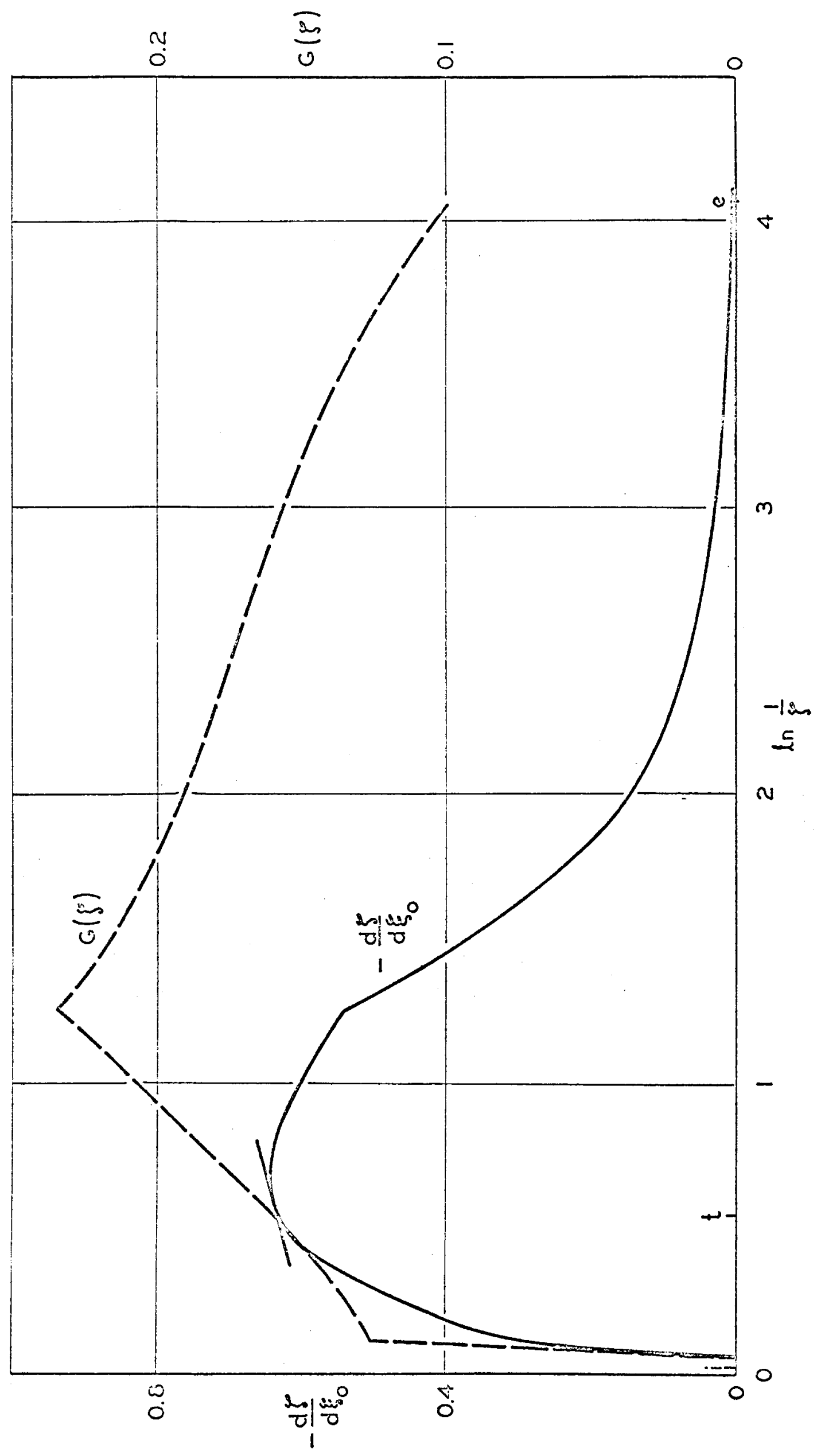

